\title{
Towards Low-Damage TEM sample preparation of Carbonaceous Materials in the Focused Ion Beam
}

\author{
Nabil D. Bassim ${ }^{1 *}$, Bradley T. De Gregorio ${ }^{1}$, A.D.L. Kilcoyne ${ }^{2}$, Keana Scott $^{3}$, Tsengming Chou ${ }^{4} \dagger$, S. \\ Wirick $^{5}$, George Cody ${ }^{6}$, Paul Fischione ${ }^{7}$, Junhai Liu ${ }^{7}$, and Rhonda M. Stroud ${ }^{1}$ \\ ${ }^{1}$ U.S. Naval Research Laboratory, Materials Science and Technology Division, Washington, DC \\ ${ }^{2}$ Advanced Light Source, Lawrence Berkeley National Laboratory, Berkeley, CA \\ ${ }^{3}$ National Institute of Standards and Technology, Gaithersburg, MD \\ ${ }^{4}$ FEI Company, Hillsboro, OR \\ ${ }^{5}$ National Synchrotron Light Source, Brookhaven National Laboratory, NY \\ ${ }^{6}$ Carnegie Institute of Washington, Washington, DC \\ ${ }^{7}$ E.A. Fischione Instruments, Inc., Export, PA \\ *U.S. Naval Research Laboratory,Code 6366, Materials Science and Technology Division, 4555 \\ Overlook Ave, SW, Washington, DC 20375 (nabil.bassim@nrl.navy.mil)
}

The Focused Ion Beam (FIB) microscope is a powerful tool for site-specific sample preparation because of its ability to sample on a micron length scale and to preserve the textural relationships between heterogeneous phases. However when working with soft (carbonaceous) materials, special care must be taken during the FIB lift-out process to preserve the sample's structural and chemical integrity. Optimization of FIB lift-out techniques for soft materials would be useful for a wide range of materials studies, from biological systems and organic electronics to remnant organic molecules contained in cosmic dust.

The degree of alteration of organic materials during the FIB lift-out process depends on both the specific type of organic material, and the details of the lift-out procedure. We used (PAAm) and Wyodak lignite coal as test materials of high and low susceptibility to damage, respectively. We prepared lift-out samples of both materials with nominal thickness $100 \mathrm{~nm}$, varying the lift-out process to determine the impact of: 1) final milling energy for polishing $(30 \mathrm{kV}-2 \mathrm{kV}), 2)$ the effect of cryo-milling, 3) use of a conductive Pt box to dissipate heat the sample 4) the effect of changing beam overlap as a heat reduction mechanism and 5) the effect of e-beam observation during milling. Thin-sections prepared by ultramicrotomy serve as a baseline of the materials in original form. To characterize the chemical bonding within the samples, we used X-ray absorption near-edge structure (XANES) spectroscopy of the carbon $\mathrm{K}$ core-loss edge. XANES studies were performed at the National Synchrotron Light Source, the Advanced Light Source and the Canadian Light Source.

Wyodak Coal: The coal samples maintain physical integrity during milling. Their spectra resemble the undamaged microtomed section, as shown in Figure 1 in peak positions though their relative intensities change. The $\pi^{*}$ peak at $285 \mathrm{eV}$ arises from aromatic bonding. The damage peak at 286.5 $\mathrm{eV}$ appears and is more pronounced in samples that have been examined with an electron beam. The peak at $288.5 \mathrm{eV}$ corresponds to the carbon-oxygen double bonds in carboxyl groups. The intensity of this peak also decreases when an electron beam has been used to observe the sample. The additional peak in the cryo sample at $\sim 291 \mathrm{eV}$ is due to heterogeneities in the coal and there is no 
suitable microtomed analogue. Coal samples retained much of their functional group chemistry through all FIB preparations.

PAAm: Samples milled at room-temperature and/ or without physical reinforcement appeared to lose mechanical integrity under the beam regardless of ion extraction voltage. Figure 2 shows dramatic changes in the shape of the C K XANES spectra for those samples. All samples that were exposed to an e-beam, e.g., monitored with "spy-mode" during milling or viewed by TEM prior to XANES measurement, show a decrease in the peak at $288 \mathrm{eV}$, which corresponds to amide functional groups $\left(\mathrm{NH}_{2}-\mathrm{C}=\mathrm{O}\right)$. A small increase in the aromatic peak at $285.0 \mathrm{eV}$ also occurs for all samples, most likely as a result of surface ion beam damage. However, samples prepared without ebeam exposure and final thinning under cryo conditions show nearly identical $\mathrm{C}$ bond distribution to the unaltered microtomed sample.

In summary, site-specific FIB-lift-out section of sensitive organic materials that approximate microtome quality in both structure and chemistry can be prepared by: (1) avoiding the standard practice in FIB-SEM instruments of using the electron beam to monitor the milling progress and (2) carrying out final thinning ( $<1$ um thick) under cryo conditions either in situ in the FIB or with a broad-beam argon ion mill.

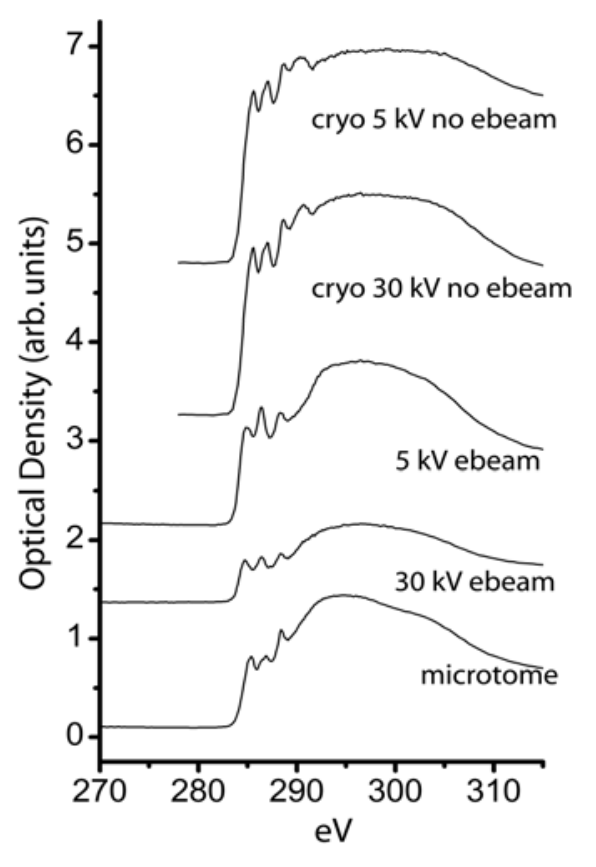

Figure 1: Structure of the C K core-loss edge from Wyodak coal samples. Changes to the relative intensities of the peaks are observed, but much of the functional chemistry is preserved under most standard milling conditions.

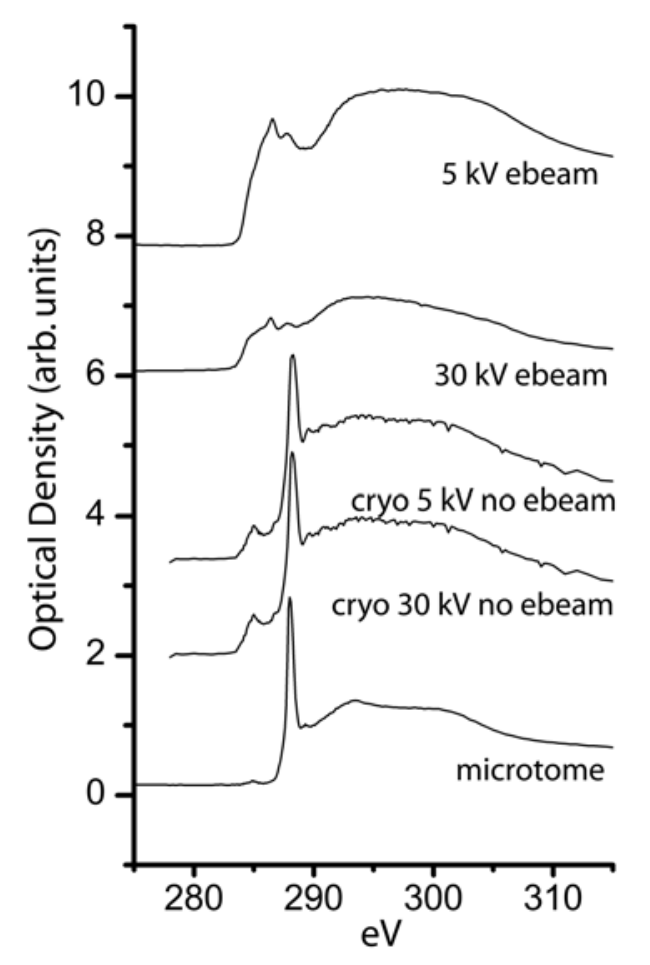

Figure 2: Structure of the C K core-loss edge from polyacrylamide (PAAm) samples. The $30 \mathrm{kV}$ FIB-prepared sample without e-beam exposure shows nearly identical $\mathrm{C}$ bonding as the mcirotomed samples. 